\title{
Family History of Stomach Cancer Unknown
}

National Cancer Institute

\section{Source}

National Cancer Institute. Family History of Stomach Cancer Unknown. NCI Thesaurus.

Code C160680.

There is an unknown family history of stomach cancer. 